\title{
How can electrode surface modification
}

\section{benefit electroanalysis?}

Eden E. L. Tanner, ${ }^{1}{ }^{2}$ and Richard G. Compton. ${ }^{1, *}$

\author{
*Corresponding author
}

Email: richard.compton@chem.ox.ac.uk

Phone: +44(0) 1865275957

Fax: $+44(0) 1865275410$

This article is dedicated with affection and admiration to Professor Joe Wang on the occasion of his 70 th birthday.

For submission to Electroanalysis

\footnotetext{
${ }^{1}$ University of Oxford, Department of Chemistry, Physical and Theoretical Chemistry Laboratory, Oxford University, South Parks Road, Oxford, OX1 3QZ, United Kingdom.

${ }^{2}$ Now at the School of Engineering \& Applied Sciences, Harvard University, Cambridge, MA, USA
} 
Surface modification of electrodes is a very popular technique touted to improve both selectivity and sensitivity of electroanalytical detection by voltammetry. In general, electrodes are evaluated both on their ability to detect the target in complex media (selectivity), and to detect a very small amount of material (sensitivity), as reflected in a low limit of detection (LoD). Note that the LoD is defined as shown in Figure 1, and often reflects a much smaller value that is realistically measurable. The method shown in Figure 1 involves the measurement of samples of a known concentration - or samples analysed by standard addition, whereby known concentrations of the analyte are added systematically to the solution. If a linear slope (b) is obtained (where $\mathrm{R}^{2}$ exceeds 0.99), verifying a linear relationship between the analytical signal and the concentration of analyte, the method is capable of detecting and quantifying the analyte of interest. A standard deviation of the slope is measured $\left(\mathrm{S}_{B}\right)$, and the LoD is calculated as $3 \mathrm{~S}_{B} / \mathrm{b}$.

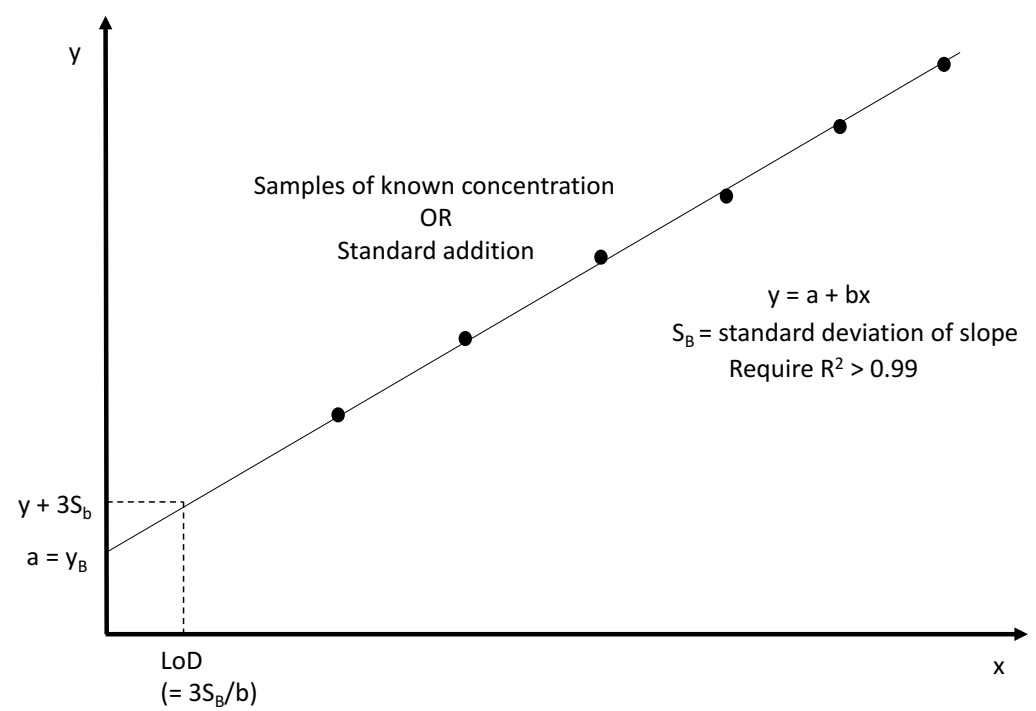

Figure 1: Calculation of the limit of detection (LoD). Adapted from Electroanalysis.[1]

Desmoni and Brunetti[2] evaluate two methods of calculating the LoD, stating that both the signal-to-noise method and the standard error method (described above) have substantial limitations, but conclude that the latter is preferable, despite the high number of theoretical conditions (13!) that are not met in practice - for example, that systematic errors must be negligible or absent, and that random errors must only occur in the y-direction and must be normally distributed. They caution the use of a LoD without a solid understanding and justification of the reported value.

The Limit of Quantification (LoQ) is an alternative value that reflects the lowest concentration measurable that reliably detects the analyte whilst meeting pre-defined goals for bias and imprecision.[3] The LoQ may be the same as the LoD, but is frequently higher (and cannot, by definition, be lower than the LoD). The LoQ is usually a much more robust value to report, although variable 'pre-defined goals' mean that a consistent understanding across the field is absent. The above is to be considered carefully when evaluating the performance of different surface modifiers in electroanalytical detection using voltammetry. 


\section{Voltammetric Techniques}

$\mathrm{Hg}^{2+}$ detection by voltammetry was recently reviewed[4] showing a vast array of modified surfaces, prompting us to ask: what do the modifications do, how do they work, and are they helpful? The electrochemical techniques used to detect mercury (which are also the mainstay techniques to detect a wide range of other heavy metals) usually involve a stripping step preceded by a preconcentration step. The preconcentration step can take the form of adsorption onto the electrode surface (Adsorptive Stripping Voltammetry, AdSV), as illustrated by the quantification of capsaicin for example in chilli peppers.[5] It can also involve an electro-deposition step of metal or metal oxides from metal ions, as in the case of Anodic Stripping Voltammetry, (ASV), used in the simultaneous detection of cadmium and lead,[6] or Cathodic Stripping Voltammetry (CSV) as exemplified in the detection of manganese.[7] These three different voltammetric techniques are illustrated in Figure 2.

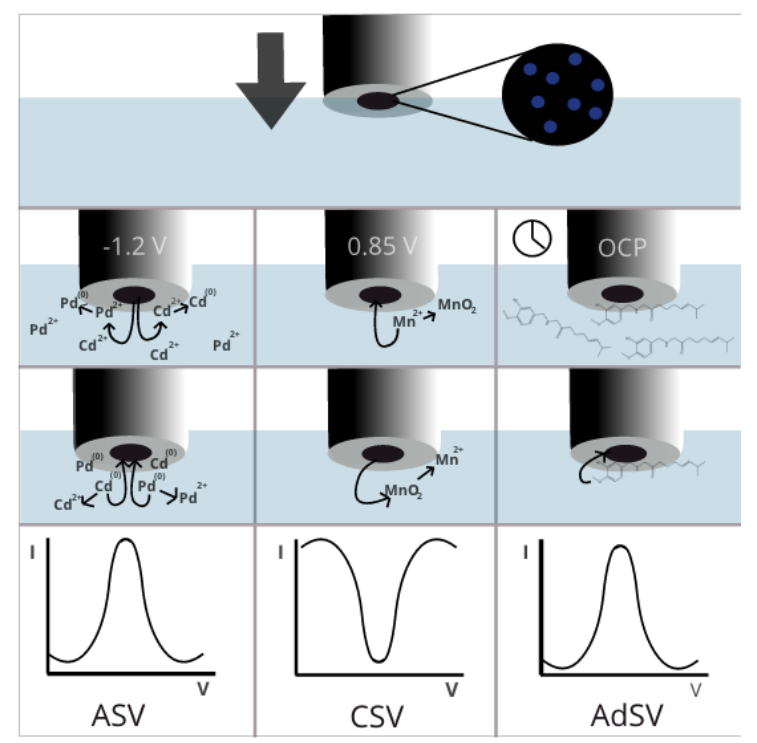

Figure 2: Schematic of Anodic Stripping Voltammetry (ASV),[6] Cathodic Stripping Voltammetry (CSV),[7] and Adsorptive Stripping Voltammetry (AdSV).[5] The arrows denote the transfer of electrons.

There are a small number of cases where voltammetry is recorded immediately after the electrode is submerged in the sample without any pre-concentration step. In these cases the apparent role of the surface modification is to switch on or accelerate the electron transfer kinetics. For example, Bhanjana et al. utilise zinc quantum dots adhered onto a gold electrode to enable detection of mercury with a detection limit of 5 ppb using Linear Sweep Voltammetry[8] with the quantum dots presumed to mediate the electron transfer. However in the overwhelming majority of electroanalytical determinations a period of accumulation is required as the signal measured directly from the solution phase analyte is either absent or tiny unless the target is present in unusually high concentrations such as the millimolar levels of glucose in blood or those of ascorbic acid in fruit juices. In the case of mercury detection, AdSV is the most popular technique, with the lowest LoDs being achieved using this method, including reported LoDs of 0.001 aM[9] for a DNA probe, and $0.1 \mathrm{nM}$ for Carbon Ionic Liquid Electrodes (CILE)[10] and Screen Printed Carbon Electrodes (SPCE).[11] ASV has been employed to report LoDs of $0.1 \mathrm{nM}$ using a gold 
nanoparticle modified indium tin oxide electrode, as illustrated in Figure 3 below.[12]

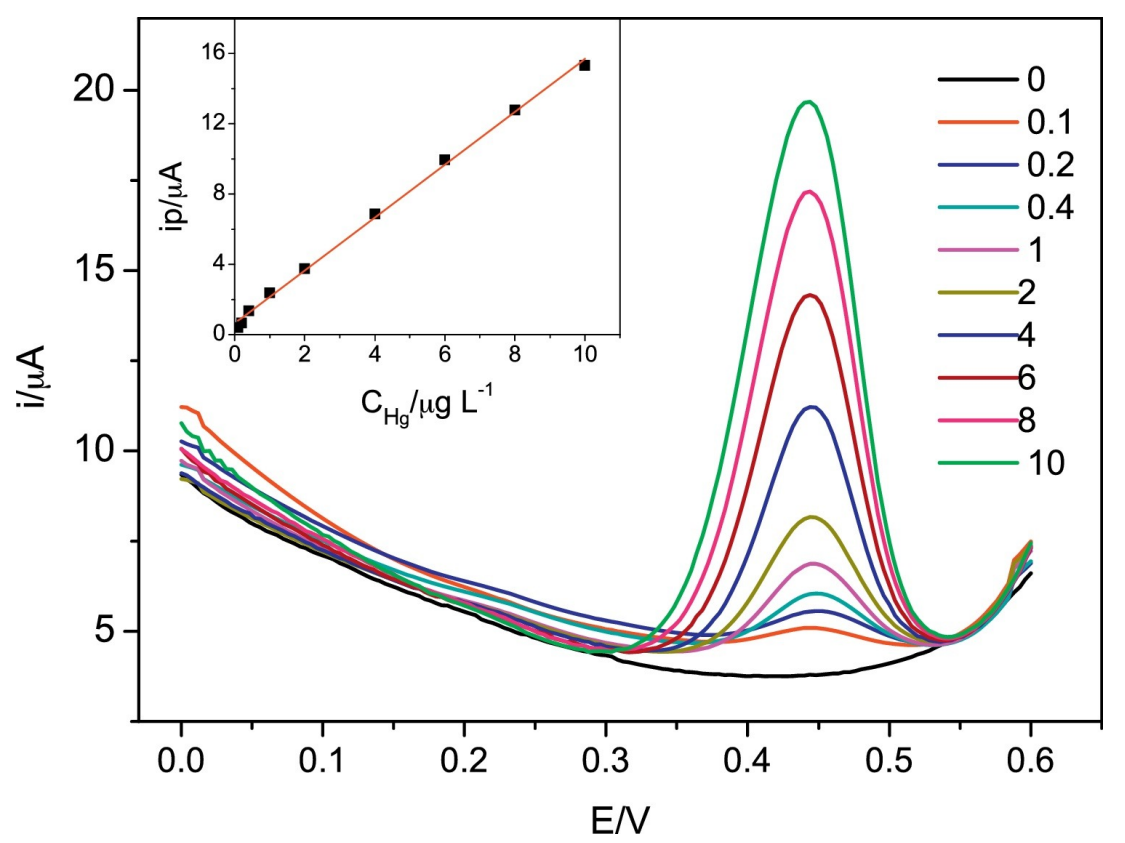

Figure 3: The response of a gold nanoparticle modified indium tin oxide electrode to low concentrations of mercury. Reprinted from Sensors and Actuators B, 220, Yuanyuan Lin, Yang Peng, and Junwei Di, Electrochemical detection of $\mathrm{Hg}$ (II) ions based on nanoporous gold nanoparticles modified indium tin oxide electrode, 1086 - 1090, Copyright (2015), with permission from Elsevier[12]

\section{Surface Modification to Influence Selectivity}

To explore the physico-chemical basis for the role of electrode modification first we consider modifying an electrode to improve its selectivity which obviously can be improved if the modified surfaces are able to bind the target but not other analytes present, enabling easier detection in authentic samples including those of environmental and medical origin. In general, if the modification possesses a chemical structure that has a strong affinity for the target to the exclusion of others it will provide an advantage in terms of selectivity. For example, in terms of inorganic mercury detection, DNA has been used to facilitate selective detection through the formation of thymine-mercury-thymine complexes, as illustrated in Figure 4 [9] whilst the strong affinity of mercury for thiol or mercaptol groups has also been exploited to enhance detection in a complicated sample matrix.[13]

Materials that show variable physico-chemical properties in the presence of different material are also utilised to generate selectivity. For example, silver nanoparticles capped with citrate molecules were shown to respond differently to a range of heavy metal ions, with their colorimetric sensitivity being driven by the formation of a metal-ligand complex.[14] In all cases increased selectivity can come at a price; complicated or lengthy modification procedures are not ideal for use in real world and high throughput applications, increasing price and risk of irreproducibility, especially if the modifiers used do not have good long term stability. In addition to these considerations, modification of the electrode to increase its selectivity confines the electrode to 

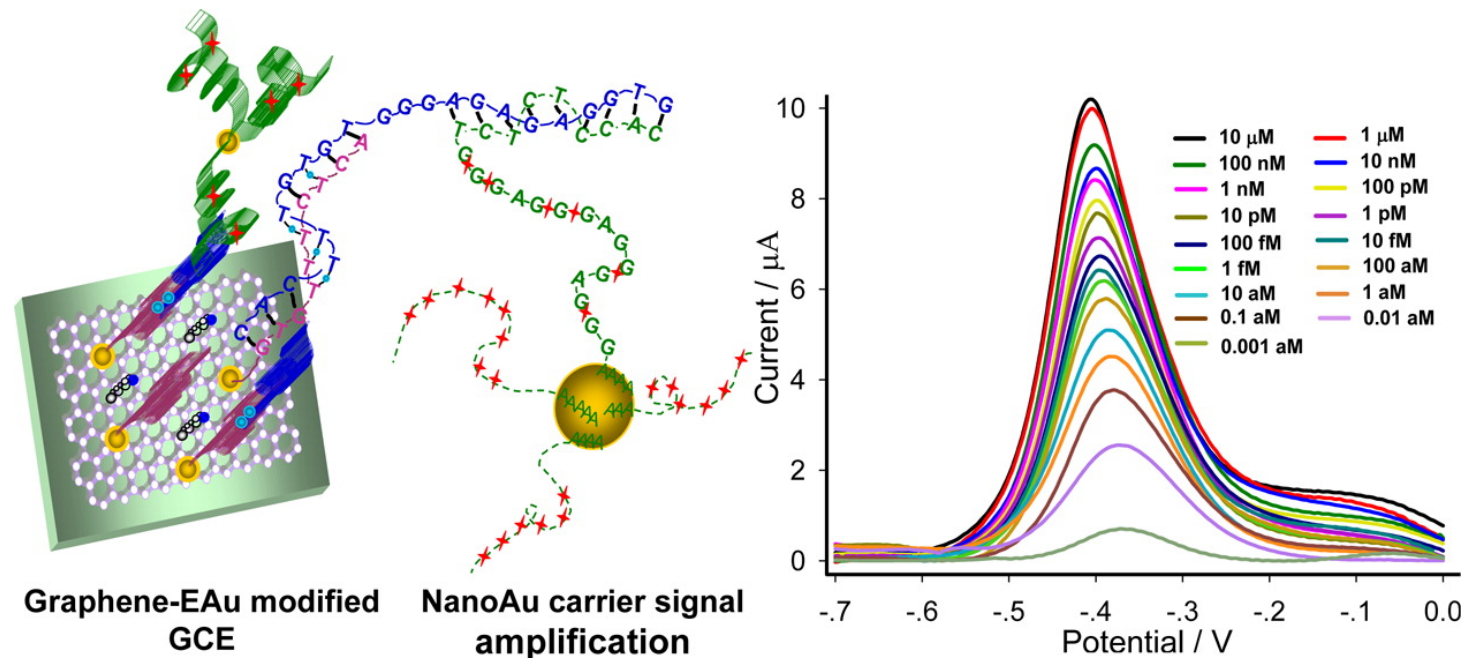

Figure 4: Illustration of a nano-gold DNA modified electrode, whereby the selective binding of the $\mathrm{Hg}^{2+}$ to the thymine enables excellent selectivity. Reprinted with permission from Electrochemical Sensor Based on Electrodeposited Graphene-Au Modified Electrode and NanoAu Carrier Amplified Signal Strategy for Attomolar Mercury Detection. Yi Zhang, Guang Ming Zeng, Lin Tang, Jun Chen, Yuan Zhu, Xiao Xiao He, and Yan He. Anal. Chem. 2015, 87 (2), 989-996. Copyright 2015 American Chemical Society.[9]

niche applications.

In the specific case of the pre-concentration of metal ions, if these are to be detected via reduction to the metal (perhaps prior to detection through ASV) then it is important to realise that the formation of the latter requires a nucleation/growth sequence and that this is not necessarily enhanced by the complexation of the ions with modifying groups on the metal surface, especially if the complexation is strong.

Next, the effect of electrode modification on possibly increasing sensitivity will be explored.

\section{The Effect of Altered Electron Transfer Kinetics}

One of the claims made about surface modification is signal enhancement through altered electron transfer kinetics. That is, if the modification alters the electron transfer kinetics for the oxidation or reduction of solution phase species such that the electrochemical process becomes less chemically irreversible, the analytical signal is amplified.

\section{Diffusion Controlled Processes}

To investigate this we first examine a diffusional response from a solution phase species and consider the equations used to describe the voltammetric peak current resulting from semi-infinite one dimensional diffusion to the electrode - the Randles-Ševčik equations.[15, 16] We consider an n electron oxidation:

$$
R e d-n e \rightleftharpoons O x
$$

First, in the case of fully reversible electron transfer on a macro electrode (where the limits of $\mathrm{k}_{0}$ are as defined by Matsuda and Ayabe[17]): 


$$
\frac{i_{p}}{\nu^{0.5}}=2.69 \times 10^{5} A D^{0.5} \mathrm{Cn}^{1.5}
$$

where $\nu$ is the scan rate, $\mathrm{A}$ is the area of the electrode, $\mathrm{D}$ is the diffusion coefficient of the analyte, $\mathrm{C}$ is the concentration (in bulk) of the analyte, and $\mathrm{n}$ is the number of electrons transferred.

The irreversible Randles-Ševčik equation describes the peak current of a voltammetric system in the event of an irreversible electrochemical rate constant[17]:

$$
\frac{i_{p}}{\nu^{0.5}}=2.99 \times 10^{5} A D^{0.5} C n\left(n^{\prime}+\beta\right)^{0.5}
$$

where $\beta$ is the transfer coefficient[18,19] of the rate determining step and $\mathrm{n}^{\prime}$ is the number of electrons transferred prior to the rate determining electron transfer.

Therefore, to consider the effect of increased irreversibility of the system on the peak current, we consider Equation 4:

$$
\frac{i_{p, \text { rev }}}{i_{p, \text { irr }}}=\frac{2.69 \times 10^{5} A D^{0.5} C \nu^{0.5} n^{1.5}}{2.99 \times 10^{5} A D^{0.5} C \nu^{0.5} n\left(n^{\prime}+\beta\right)^{0.5}}=0.9 \times \frac{n^{0.5}}{\left(n^{\prime}+\beta\right)^{0.5}}
$$

Kozub et al. [20] considered this in detail for a one electron transfer, and concluded that the maximum enhancement of the current was a factor of just 1.27 which represents a relatively small increase in the current signal for the effort invested in modifying an electrode. Indeed Kozub accordingly recommended the use of bare glassy carbon electrodes for the oxidation of nitrite over and above a diversity of chemically modified alternatives.

On the other hand changing the electrochemical rate constant $\mathrm{k}_{0}$ can have a large effect on peak potential, and so sometimes be helpful in a situation where several components of a mixture have nearly identical peak potentials so that there voltammetric responses overlap heavily. Altering (increasing and/or decreasing) the electrochemical reversibility of one or more of the components of the mixture can assist in isolating a particular electrochemical event for analytical purposes.

Many analytes of interest undergo electrochemical oxidation or reduction with $\mathrm{n}>1$. Thus Table 1 shows how the enhancement factor given in equation 3 varies as the total number of electrons, $\mathrm{n}$, transferred overall in the electrode process increases. Here we assume $\beta$ is 0.5 for the case where the first electron transfer is rate determining so that $\mathrm{n}^{\prime}$ is 0 . If $\mathrm{n}^{\prime}$ is larger than zero then the enhancement factor will be lower.

\begin{tabular}{cc} 
Number of Electrons & Factor Difference \\
\hline 1 & 1.27 \\
2 & 1.80 \\
3 & 2.20 \\
4 & 2.55 \\
\hline
\end{tabular}

Table 1: Factor difference as $\mathrm{n}$ changes, where $\mathrm{n}^{\prime}=0$, and $\beta=0.5$ for a diffusion controlled signal. 
Table 1 shows clearly that when a relatively small number of electrons are transferred, a change in the kinetics is unlikely to result in a usefully significant enhancement in the peak current. However, in systems whereby a large number of electrons might be expected to transfer glucose oxidation, for example - changing the kinetics may provide a helpful enhancement in the peak current, especially if the rate determining step is the first electron transfer. However, for most heavy metal ions, the numbers of electrons transferred is unlikely to exceed two or three, and so modification of the electrode to elicit an electrochemically reversible process will not improve sensitivity especially in comparison with either pre-concentration techniques such as ASV.

\section{Adsorbed Species}

In the case that the analytical voltammetric signal relates to an adsorbed species then the enhancement factor associated with the transition from irreversible to reversible electrode kinetics can be estimated from the following equations.

First, in the case of reversible electrode kinetics, the peak current is described thus:

$$
i_{p}=\frac{n^{2} F^{2}}{4 R T} \nu A \Gamma_{0}^{*}
$$

where $\Gamma_{0}^{*}$ is the amount of material adsorbed on the electrode and all other symbols are as defined above.

Second, the peak current in the case of irreversible electrode kinetics is described as follows:

$$
i_{p}=n \frac{\left(n^{\prime}+\beta\right) F^{2} \nu A \Gamma_{0}^{*}}{2.718 R T}
$$

Finally, to consider the affect of increasing the irreversibility of the system of the peak current, we use the following equation.

$$
\frac{i_{p, r e v}}{i_{p, i r r}}=\frac{2.718 n}{4\left(n^{\prime}+\beta\right)}
$$

From Equation 7, we can calculate the expected factor difference if a process were to change from electrode kinetics that are fully reversible to fully irreversible (once again assuming $\beta$ is 0.5 and $\left.\mathrm{n}^{\prime}=0\right)$.

\begin{tabular}{cc} 
Number of Electrons & Factor Difference \\
\hline 1 & 1.36 \\
2 & 2.72 \\
3 & 4.08 \\
4 & 5.44 \\
\hline
\end{tabular}

Table 2: Factor difference as n changes, where $\mathrm{n}^{\prime}=0$, and $\beta=0.5$ for an adsorption controlled signal.

In contrast with 1 , the effect of altering the kinetics on the observed peak current is much greater for a process governed by adsorption, as shown in 2 , than a diffusionally controlled process. 
For a 2 or 3 electron process, one might achieve a 3 - 4 fold enhancement in the peak current if the detection relies on an adsorbed species.

\section{A Note on More Sophisticated Voltammetry}

A further consideration relates to the use of more sophisticated forms of voltammetry than linear sweep or cyclic voltammetry - most notably pulse voltammetry, or the now popular square wave voltammetry (SWV). Of particular importance is the analysis of the response of surface bound processes which shows[21] that in the case of fast charge transfers, negligible currents are obtained for the application of potential pulses. Thus as the electrochemical rate constant for the charge transfer increases from slow to fast, the peak current passes through a maximum. It follows that any surface modification must seek to provide catalysis matching the optimal rate constant, an exercise which is likely to be amenable to a combination of empirical optimization and a large element of good luck!

\section{Increased Surface Area}

Another way in which modification is often claimed to increase sensitivity is through increased electrode surface area. This is frequently cited as a reason for enhanced analytical signals, particularly when using nanomaterials. However, not all situations necessarily benefit from an increase in surface area and it is important to understand the physical basis of the analytical procedure before the role of surface area and any benefits can be assessed.

For example, in the case of AdSV, increased surface area can in principle improve sensitivity by providing additional sites for the target to bind in the pre-concentration step. However if the rate of adsorption on the electrode surface is relatively fast such that the adsorption is effectively diffusion controlled then the overall rate of analyte accumulation reflects the mass transport to the geometric area of the electrode rather than the true surface area. Thus changing the electrode area under conditions of fast adsorption is of little value. Indeed thought suggests that there is an optimum rate of adsorption for the pre-concentration step if the electrode surface is to be manipulated so as to increase its surface area - too slow an adsorption rate results in negligible build-up of analyte whereas too fast results in the potential benefits of the increased true surface area being masked by diffusion control.

It is also essential to consider the mechanism by which the accumulated material subsequently undergoes electron transfer in the measurement step of the AdsV procedure. Two limiting cases can be envisaged. In the first the accumulated material is directly oxidized or reduced in its adsorbed state - for example in the cases of capsaicin[5] and caffeine[22] - whereas in the second the material has to first desorb and then transport to the electrode surface. The latter case corresponds to the now common practice of using insulating particles (imprinted polymeric nanobeads in the detection of mercury, for example[10]) and materials as electrode modifiers. Recent theoretical analysis[23] has shown that the response in this second case is highly sensitive to the thermodynamics and kinetics of the adsorption step - too strong an adsorption kills the analytical signal whereas a weak adsorption offers too little pre-concentration so that again a fine balance operates and this yet again makes the a priori design of the electrode surfaces challenging. In any case, under this second scenario it was concluded that the electrode modification was unlikely to realize more than an order of magnitude improvement unless the surface coverages associated with the adsorption could be increased above typical monolayer values. In general it seems preferable to modify an electrode with conductive particles rather than insulators unless the latter can conduct charge (via electron hopping) over the insulator surface so as to allow the oxidation/reduction of the adsorbed pre-concentrated material without the need for desorption. 
Such surface conductivity has recently been demonstrated for the case of catechol adsorbed on particles of alumina.[24] In the context of the analysis of solution phase species electrode porosity generated by the surface modification of an electrode with conductive materials can give rise to useful current enhancements. These may be rather larger in magnitude than those described above for semi-infinite diffusion to a flat, planar electrode. In experimental practice for example the porosity can be generated on the electrode from layers of nanoparticles[25], of carbon nanotubes[26, 27] or dispersed metal particles.[28] The increased currents observed together with the shift of electrochemically irreversible waves to lower overpotential can be explained by the change of mass transport regime from semi-infinite diffusion to that associated with that of thin layer diffusion.[26, 29] In this situation the soluble electroactive species ?trapped? within the surface pores of the modified electrode plays a dominant role in the observed voltammetric response. Note that the improved response can be due to the change on mass transport regime alone; no electro-catalysis is essential. Distinguishing between the two explanations in any situation is powerfully, clearly and easily accomplished by means of either differential pulse or square wave voltammetry.[30] Note that the shifts in the potentials at which the voltammetric signals from different species can be observed can be useful in the analysis of mixtures of different species which have overlapping responses under conditions of semi-infinite diffusion.[31] The deliberate, intelligent use of surface modification of electrodes by conductive layers for enhanced electro-analysis has been demonstrated elegantly and effectively for the case of screen printed electrodes by Mendez and co-workers.[32]

\section{Conclusions}

In conclusion, electrode modification can serve to increase the selectivity and sensitivity of electroanalytical protocols, but it is important that it is accompanied by careful considerations of precisely what the modification seeks to achieve and the associated physico-chemical mechanisms. Otherwise, extremely complicated and delicate modifications will continue to abound but with very little hope of being deployable in practical applications, and with little evidence that the often laborious and expensive modifications provide a justifiable pay back in terms of enhancement of the performance of the sensor.

\section{Acknowledgements}

The authors thank Alex L. Suherman for thought provoking discussions. The research leading to these results has received partial funding from the European Research Council under the European Union's Seventh Framework Programme (FP/2007-2013)/ERC Grant Agreement no. [320403].

\section{References}

1. C. M. A. Brett, A. M. O. Brett, Electroanalysis, Oxford University Press, 2005.

2. E Desimoni, B Brunetti, "About estimating the limit of detection by the signal to noise approach", Pharm. Anal. Acta 2015, 6, 1.

3. D. A. Armbruster, T. Pry, "Limit of blank, limit of detection and limit of quantitation", Clin. Biochem. Rev. 2008, 29, S49.

4. A. L. Suherman, E. E. L. Tanner, R. G. Compton, "Recent developments in inorganic $\mathrm{Hg}^{2+}$ detection by voltammetry", Trends Anal. Chem.; TrAC 2017, 94, 161. 
5. R. T. Kachoosangi, G. G. Wildgoose, R. G. Compton, "Carbon nanotube-based electrochemical sensors for quantifying the 'heat' of chilli peppers: the adsorptive stripping voltammetric determination of capsaicin", Analyst 2008, 133, 888, DOI 10.1039/B803588A.

6. K. E. Toghill, G. G. Wildgoose, A. Moshar, C. Mulcahy, R. G. Compton, "The Fabrication and Characterization of a Bismuth Nanoparticle Modified Boron Doped Diamond Electrode and Its Application to the Simultaneous Determination of Cadmium(II) And Lead(II)", Electroanal. 2008, 20, 1731, DOI 10.1002/elan.200804277.

7. A. J. Saterlay, J. S. Foord, R. G. Compton, "Sono-cathodic stripping voltammetry of manganese at a polished boron-doped diamond electrode: application to the determination of manganese in instant tea", Analyst 1999, 124, 1791, DOI 10.1039/A906851A.

8. G. Bhanjana, N. Dilbaghi, R. Kumar, S. Kumar, "Zinc Oxide Quantum Dots as Efficient Electron Mediator for Ultrasensitive and Selective Electrochemical Sensing of Mercury", Electrochim. Acta 2015, 178, 361, DOI https://doi.org/10.1016/j. electacta.2015. 07.113.

9. Y. Zhang, G. M. Zeng, L. Tang, J. Chen, Y. Zhu, X. X. He, Y. He, "Electrochemical Sensor Based on Electrodeposited Graphene-Au Modified Electrode and NanoAu Carrier Amplified Signal Strategy for Attomolar Mercury Detection", Anal. Chem. 2015, 87, 989, DOI 10.1021/ac503472p.

10. A. Bahrami, A. Besharati-Seidani, A. Abbaspour, M. Shamsipur, "A highly selective voltammetric sensor for nanomolar detection of mercury ions using a carbon ionic liquid paste electrode impregnated with novel ion imprinted polymeric nanobeads", Mat. Sci. Eng. C 2015, 48, 205, DOI https://doi.org/10.1016/j.msec.2014.12.005.

11. M. Armas, R. María-Hormigos, A. Cantalapiedra, M. Gismera, M. Sevilla, J. Procopio, "Multiparametric optimization of a new high-sensitive and disposable mercury (II) electrochemical sensor", Anal. Chim. Acta 2016, 904, 76, DOI https://doi.org/10.1016/j.aca. 2015.11.016

12. Y. Lin, Y. Peng, J. Di, "Electrochemical detection of $\mathrm{Hg}(\mathrm{II})$ ions based on nanoporous gold nanoparticles modified indium tin oxide electrode", Sens. Actuators B Chem. 2015, 220, 1086, DOI https://doi.org/10.1016/j.snb.2015.06.064.

13. S. Sahoo, A. K. Satpati, A. V. R. Reddy, "Modification of gold electrode by self-assembled 2-mercapto benzothiazole for the determination of $\mathrm{Hg}^{2+}$ ", Int. J. Environ. Anal. Chem. 2014, 94, 1050, DOI 10.1080/03067319.2014.914186.

14. K. Ngamchuea, C. Batchelor-McAuley, S. V. Sokolov, R. G. Compton, "Dynamics of Silver Nanoparticles in Aqueous Solution in the Presence of Metal Ions", Anal Chem. 2017, 89, PMID: 28882041, 10208, DOI 10.1021/acs . analchem.7b01470.

15. J. Randles, "A cathode ray polarograph. II. The current voltage curves", Trans. Faraday Soc. 1948, 44, 327 .

16. A Ševčik, "Oscillographic polarography with periodical triangular voltage", Collect. Czech. Chem. Commun. 1948, 13, 349.

17. H. Matsuda, Y. Ayabe, "Zur Theorie der Randles-Sevčikschen Kathodenstrahl-Polarographie", Ber. Bunsenges. Phys. Chem. 1955, 59, 494.

18. R. Guidelli, R. G. Compton, J. M. Feliu, E. Gileadi, J. Lipkowski, W. Schmickler, S. Trasatti, "Defining the transfer coefficient in electrochemistry: An assessment (IUPAC Technical Report)", Pure Appl. Chem. 2014, 86, 245. 
19. R. Guidelli, R. G. Compton, J. M. Feliu, E. Gileadi, J. Lipkowski, W. Schmickler, S. Trasatti, "Definition of the transfer coefficient in electrochemistry (IUPAC Recommendations 2014)", Pure Appl. Chem. 2014, 86, 259.

20. B. R. Kozub, N. V. Rees, R. G. Compton, "Electrochemical determination of nitrite at a bare glassy carbon electrode; why chemically modify electrodes?", Sens. Actuators B Chem. 2010, 143,539 .

21. A Molina, J. González, "Pulse voltammetry in physical electrochemistry and electroanalysis", Monographs in Electrochemistry 2016.

22. N. Lezi, S. Economopoulos, M. Prodromidis, A. Economou, N. Tagmatarchis, "Fabrication of a "Green' and Low-Cost Screen-Printed Graphene Sensor and Its Application to the Determination of Caffeine by Adsorptive Stripping Voltammetry", Int. J. Electrochem. Sci. 2017, 12, 6054 .

23. H. T. Chan, E. Kätelhön, R. G. Compton, "Voltammetry at electrodes decorated with an insulating porous film: Understanding the effects of adsorption", J. Electroanal. Chem. 2017, 801,135 .

24. Q. Lin, R. G. Compton, "Impacts reveal and quantify monolayer adsorption on single alumina particles", Russ. J. Electrochem. 2017, 53, 994.

25. X. Huang, Y. Li, Y. Chen, L. Wang, "Electrochemical determination of nitrite and iodate by use of gold nanoparticles/poly (3-methylthiophene) composites coated glassy carbon electrode", Sens. Actuator B-Chem. 2008, 134, 780.

26. I. Streeter, G. G. Wildgoose, L. Shao, R. G. Compton, "Cyclic voltammetry on electrode surfaces covered with porous layers: an analysis of electron transfer kinetics at single-walled carbon nanotube modified electrodes", Sens. Actuator B-Chem. 2008, 133, 462.

27. M. J. Sims, N. V. Rees, E. J. Dickinson, R. G. Compton, "Effects of thin-layer diffusion in the electrochemical detection of nicotine on basal plane pyrolytic graphite (BPPG) electrodes modified with layers of multi-walled carbon nanotubes (MWCNT-BPPG)", Sens. Actuator B-Chem. 2010, 144, 153.

28. I. G. Casella, A. M. Salvi, "Voltammetric behavior and ion chromatographic detection of nitrite at a dispersed platinum glassy carbon electrode", Electroanal. 1997, 9, 596.

29. G. P. Keeley, M. E. Lyons, "The effects of thin layer diffusion at glassy carbon electrodes modified with porous films of single-walled carbon nanotubes", Int. J. Electrochem. Sci 2009, 4, 794 .

30. E. Laborda, A. Molina, F. Martínez-Ortiz, R. G. Compton, "Electrode modification using porous layers. Maximising the analytical response by choosing the most suitable voltammetry: Differential Pulse vs Square Wave vs Linear sweep voltammetry", Electrochim. Acta 2012, 73,3 .

31. M. C. Henstridge, E. J. Dickinson, M. Aslanoglu, C. Batchelor-McAuley, R. G. Compton, "Voltammetric selectivity conferred by the modification of electrodes using conductive porous layers or films: The oxidation of dopamine on glassy carbon electrodes modified with multiwalled carbon nanotubes", Sens. Actuator B-Chem. 2010, 145, 417.

32. S Botasini, A. Martí, E Méndez, "Thin-layer voltammetry of soluble species on screen-printed electrodes: proof of concept", Analyst 2016, 141, 5996. 